\title{
Spectral expressions for modelling the gravitational field of the Earth's crust density structure
}

\author{
Robert Tenzer ${ }^{1}$, PAvel NovÁK ${ }^{2}$, HAMAyun $^{3}$ AND Peter VAJdA ${ }^{4}$ \\ 1 National School of Surveying, Faculty of Sciences, University of Otago, Dunedin, New \\ Zealand (robert.tenzer@surveying.otago.ac.nz) \\ 2 Department of Mathematics, University of West Bohemia, Univerzitní 22, 30614 Plzeň, \\ Czech Republic \\ 3 Delft Institute of Earth Observation and Space Systems (DEOS), TU Delft, Kluyverweg 1, \\ Delft, The Netherlands \\ 4 Geophysical Institute, Slovak Academy of Sciences, Dúbravská cesta 9, 84528 Bratislava, \\ Slovak Republic
}

Received: March 8, 2011; Revised: May 9, 2011; Accepted: May 25, 2011

\begin{abstract}
We derive expressions for computing the gravitational field (potential and its radial derivative) generated by an arbitrary homogeneous or laterally varying density contrast layer with a variable depth and thickness based on methods for a spherical harmonic analysis and synthesis of gravity field. The newly derived expressions are utilised in the gravimetric forward modelling of major known density structures within the Earth's crust (excluding the ocean density contrast) beneath the geoid surface. The gravitational field quantities due to the sediments and crust components density contrasts, shown in numerical examples, are computed using the $2 \times 2$ arc-deg discrete data from the global crustal model CRUST2.0. These density contrasts are defined relative to the adopted value of the reference crustal density of $2670 \mathrm{kgm}^{-3}$. All computations are realised globally on a $1 \times 1$ arc-deg geographical grid at the Earth's surface. The maxima of the gravitational signal due to the sediments density contrast are mainly along continental shelf regions with the largest sedimentary deposits. The corresponding maxima due to the consolidated crust components density contrast are over areas of the largest continental crustal thickness with variable geological structure.
\end{abstract}

Keywords: density, Earth's crust, forward modelling, gravity, spherical harmonics

\section{INTRODUCTION}

In geophysical studies investigating the lithosphere structure the gravitational effect of the known subsurface mass density distribution is modelled and subsequently removed from observed gravity in order to reveal the remaining gravitational signal of the unknown anomalous subsurface density distribution or the density interface. The strongest signal to be subtracted from observed gravity is due to the topography (onshore) and the bathymetry (offshore). The currently available global geopotential models and the global 
elevation and bathymetry data allow modelling the topography-corrected and bathymetrystripped gravity field quantities to a very high spectral resolution (up to spherical harmonic degree 2160) using methods for a spherical harmonic analysis and synthesis of gravitational field. The next strongest signal in gravity data is due to the crustal/lithospheric thickness and density composition as a result of the combination of its isostatic and tectono-physical states (Tenzer et al., 2009). An isostatic compensation scheme may be adopted to compute the isostatic correction to gravity data, or a crustal model is used to compute the crustal components stripping corrections. In this latter step various methods have been applied depending on the purpose of the study (for the literature overview of global and regional studies we refer readers to Tenzer et al., 2009). In regional studies the stripped gravity data are typically interpreted by an integrated forward modelling with the use of all possible geophysical constraints. For global studies the best currently available global crustal model is CRUST2.0 (Bassin et al., 2000), which is an upgrade of CRUST5.1 (Mooney et al., 1998). The publically available CRUST2.0 model contains information on the crustal thickness and the subsurface spatial distribution and density of the following global components: ice; ocean; soft and hard sediments; upper, middle, and lower (consolidated) crust. Čadek and Martinec (1991) were first who computed globally the Earth's crustal thickness model complete to the spherical harmonic degree 30. It is worth noting that the information about the crustal structure is not incorporated in the global models of the crust thickness CUB2 (Shapiro and Ritzwoller, 2002) and MDN (Meier et al., 2007), both compiled based on the seismic data analysis.

Various expressions in terms of spherical harmonics were derived and applied to compute the topographic and crust density contrast stripping gravity corrections assuming the homogeneous mass density distribution. A change of atmospheric density with elevation was assumed in computing the atmospheric gravitational effects, for instance, by Sjöberg and Nahavandchi (2000). Tenzer et al. (2011) facilitated a depth-depended seawater density model in computing the bathymetric stripping gravity corrections. Tenzer et al. (2010) derived expressions for computing the ice density contrast stripping corrections to gravity field in terms of spherical harmonics. A more generalised form of spectral expressions which takes into account the lateral density distribution was presented by Sjöberg (1998) and others; see also a more recent study by Eshagh (2009). In this study, we derive the expressions for computing the gravitational field generated by the laterally varying or homogeneous mass density contrast layer with a variable depth and thickness in terms of spherical harmonics. Disregarding depth-dependent density variations (for instance in sedimentary basins due to compaction, cf. Artemjev et al., 1994), these expressions allow the fast and effective gravimetric forward modelling of density contrasts within the Earth's solid crust (i.e., excluding the ocean density contrast) based on currently available global crustal models. These expressions are derived in Section 2. The numerical examples are presented and discussed in Section 3. The summary and conclusions are given in Section 4. 


\section{GRAVITATIONAL FIELD OF AN ARBITRARY LATERALLY VARYING DENSITY CONTRAST LAYER}

In spherical approximation, the gravitational potential $V$ generated by the laterally varying density contrast layer with a variable depth and thickness computed at a position $(r, \Omega)$ is defined by the following spatial representation of Newton's volume integral

$$
V(r, \Omega)=G \iint_{\Phi} \Delta \rho\left(\Omega^{\prime}\right) \int_{R-D_{L}\left(\Omega^{\prime}\right)}^{R-D_{U}\left(\Omega^{\prime}\right)} \ell^{-1}\left(r, \psi, r^{\prime}\right) r^{\prime 2} \mathrm{~d} r^{\prime} d \Omega^{\prime},
$$

where $G=6.674 \times 10^{-11} \mathrm{~m}^{3} \mathrm{~kg}^{-1} \mathrm{~s}^{-2}$ is Newton's gravitational constant; $R=6371 \times 10^{3} \mathrm{~m}$ is the Earth's mean radius (which approximates the geocentric radius of the geoid surface); $D_{U}$ and $D_{L}$ are the depths (reckoned relative to the sphere of radius $R$ ) of the upper and lower bounds of the volumetric layer, respectively; $\ell$ is the Euclidean spatial distance between positions of the computation point $(r, \Omega)$ and the integration (running) point $\left(r^{\prime}, \Omega^{\prime}\right)$, and $\psi$ is the respective spherical distance; $d \Omega^{\prime}=\sin \phi^{\prime} d \phi^{\prime} d \lambda^{\prime}$ is the infinitesimal surface element of the unit sphere; and the full spatial angle is denoted as $\Phi=\left\{\Omega^{\prime}=\left(\phi^{\prime}, \lambda^{\prime}\right): \phi^{\prime} \in[-\pi / 2, \pi / 2] \wedge \lambda^{\prime} \in[0,2 \pi)\right\}$. The 3D position is defined in geocentric spherical coordinates $(r, \phi, \lambda)$, where $r$ is the geocentric radius and $\Omega=(\phi, \lambda)$ denotes the geocentric direction with the geocentric spherical latitude $\phi$ and longitude $\lambda$. The laterally varying density contrast $\Delta \rho$ in Eq.(1) is defined as the difference between the reference (constant) density of the Earth's crust $\rho^{\text {crust }}$ and the laterally varying density $\rho$ within the volumetric layer, i.e.

$$
\Delta \rho\left(\Omega^{\prime}\right)=\rho^{\text {crust }}-\rho\left(\Omega^{\prime}\right), \quad\left[R-D_{U}\left(\Omega^{\prime}\right) \geq r^{\prime} \geq R-D_{L}\left(\Omega^{\prime}\right): \Omega^{\prime} \in \Phi\right] .
$$

To derive the expression for the gravitational potential $V$ in the spectral representation, Eq.(1) is first rewritten as

$$
\begin{gathered}
V(r, \Omega)=G \iint_{\Phi} \Delta \rho\left(\Omega^{\prime}\right) \int_{R-D_{L}\left(\Omega^{\prime}\right)}^{R} \ell^{-1}\left(r, \psi, r^{\prime}\right) r^{\prime 2} \mathrm{~d} r^{\prime} d \Omega^{\prime} \\
-G \iint_{\Phi} \Delta \rho\left(\Omega^{\prime}\right) \int_{R-D_{U}\left(\Omega^{\prime}\right)}^{R} \ell^{-1}\left(r, \psi, r^{\prime}\right) r^{\prime 2} \mathrm{~d} r^{\prime} d \Omega^{\prime} .
\end{gathered}
$$

The first constituent on the right-hand side of Eq.(3) is the gravitational contribution generated by the volumetric mass of laterally varying density contrast enclosed between the lower bound $\left\{D_{L}\left(\Omega^{\prime}\right): \Omega^{\prime} \in \Phi\right\}$ and the reference sphere of radius $R$. The second constituent represents the gravitational contribution generated by the volumetric mass of laterally varying density contrast enclosed between the upper bound $\left\{D_{U}\left(\Omega^{\prime}\right): \Omega^{\prime} \in \Phi\right\}$ and the reference sphere of radius $R$. 
The spectral representation of the reciprocal spatial distance $\ell^{-1}$ for the external convergence domain $r \geq r^{\prime} \quad\left(r \geq R \wedge r^{\prime} \leq R\right)$ is given by (e.g., Hobson, 1931)

$$
\ell^{-1}\left(r, \psi, r^{\prime}\right)=\frac{1}{r} \sum_{n=0}^{\infty}\left(\frac{r^{\prime}}{r}\right)^{n} P_{n}(\cos \psi),
$$

where $P_{n}$ are the Legendre polynomials of degree $n$ for the argument of cosine of the spherical distance $\psi$. The series in Eq.(4) is uniformly convergent for $r \geq r^{\prime}$. Substituting the fundamental harmonic function in Eq.(4) to Eq.(3), we arrive at

$$
\begin{gathered}
V(r, \Omega)=G \sum_{n=0}^{\infty}\left(\frac{1}{r}\right)^{n+1} \iint_{\Phi} \Delta \rho\left(\Omega^{\prime}\right) P_{n}(\cos \psi) \int_{R-D_{L}\left(\Omega^{\prime}\right)}^{R} r^{\prime n+2} \mathrm{~d} r^{\prime} d \Omega^{\prime} \\
-G \sum_{n=0}^{\infty}\left(\frac{1}{r}\right)^{n+1} \iint_{\Phi} \Delta \rho\left(\Omega^{\prime}\right) P_{n}(\cos \psi) \int_{R-D_{U}\left(\Omega^{\prime}\right)}^{R} r^{\prime n+2} \mathrm{~d} r^{\prime} d \Omega^{\prime} .
\end{gathered}
$$

Since the expansion of Newton's integral kernel converges uniformly when computed at locations outside the gravitating masses, the interchange of summation and integration in Eq.(5) is permissible (cf. Moritz, 1980). The application of the binomial theorem to the term $r^{\prime n+2}$ in Eq.(5) yields

$$
r^{\prime n+2}=R^{n+2}\left(1-\frac{R-r^{\prime}}{R}\right)^{n+2} \cong R^{n+2} \sum_{k=0}^{n+2}\left(\begin{array}{c}
n+2 \\
k
\end{array}\right)\left(\frac{R-r^{\prime}}{R}\right)^{k}(-1)^{k} .
$$

From Eq.(6), the solution to the radial integral in the first constituent on the right-hand side of Eq.(5) is found to be

$$
\begin{gathered}
\int_{R-D_{L}\left(\Omega^{\prime}\right)}^{R} r^{\prime n+2} \mathrm{~d} r^{\prime} \cong R^{n+2} \int_{R-D_{L}\left(\Omega^{\prime}\right)}^{R} \sum_{k=0}^{n+2}\left(\begin{array}{c}
n+2 \\
k
\end{array}\right)\left(\frac{R-r^{\prime}}{R}\right)^{k}(-1)^{k} \mathrm{~d} r^{\prime} \\
=R^{n+3} \sum_{k=0}^{n+2}\left(\begin{array}{c}
n+2 \\
k
\end{array}\right)\left[\frac{D_{L}\left(\Omega^{\prime}\right)}{R}\right]^{k+1} \frac{(-1)^{k}}{k+1} .
\end{gathered}
$$

By analogy with Eq.(7), the radial integral in the second constituent on the right-hand side of Eq.(5) is defined as

$$
\begin{gathered}
\int_{R-D_{U}\left(\Omega^{\prime}\right)}^{R} r^{\prime n+2} \mathrm{~d} r^{\prime} \cong R^{n+2} \int_{R-D_{U}\left(\Omega^{\prime}\right)}^{R} \sum_{k=0}^{n+2}\left(\begin{array}{c}
n+2 \\
k
\end{array}\right)\left(\frac{R-r^{\prime}}{R}\right)^{k}(-1)^{k} \mathrm{~d} r^{\prime} \\
=R^{n+3} \sum_{k=0}^{n+2}\left(\begin{array}{c}
n+2 \\
k
\end{array}\right)\left[\frac{D_{U}\left(\Omega^{\prime}\right)}{R}\right]^{k+1} \frac{(-1)^{k}}{k+1} .
\end{gathered}
$$

Our numerical analysis of the convergence domain revealed that the first five terms of the binomial series multiplied by $R^{n+3}$ approximate the radial integrals on the left-hand 
side of Eqs.(7) and (8) with a relative accuracy better than $0.3 \%$ (for maximum depths of the Moho density interface less than $100 \times 10^{3} \mathrm{~m}$, and the spectral resolution complete to degree $\bar{n}=180$ of spherical harmonics), which is below the relative inaccuracy due to the spherical approximation. When increasing the maximum degree of spherical harmonics above this limit, the series eventually becomes divergent. The analysis of the convergence domain is thus essential for finding an optimal truncation degree depending on the required numerical accuracy. The convergence and optimal truncation of binomial series were studied in detail by Rummel et al. (1988) and Sun and Sjöberg (2001). The substitution from Eqs.(7) and (8) to Eq.(5) yields

$$
\begin{aligned}
& V(r, \Omega)=G R^{2} \sum_{n=0}^{\infty}\left(\frac{R}{r}\right)^{n+1} \sum_{k=0}^{n+2}\left(\begin{array}{c}
n+2 \\
k
\end{array}\right)\left(\frac{1}{R}\right)^{k+1} \frac{(-1)^{k}}{k+1} \\
& \times \iint_{\Phi} \Delta \rho\left(\Omega^{\prime}\right) D_{L}^{k+1}\left(\Omega^{\prime}\right) P_{n}(\cos \psi) d \Omega^{\prime} \\
&-G R^{2} \sum_{n=0}^{\infty}\left(\frac{R}{r}\right)^{n+1} \sum_{k=0}^{n+2}\left(\begin{array}{c}
n+2 \\
k
\end{array}\right)\left(\frac{1}{R}\right)^{k+1} \frac{(-1)^{k}}{k+1} \\
& \quad \times \iint_{\Phi} \Delta \rho\left(\Omega^{\prime}\right) D_{U}^{k+1}\left(\Omega^{\prime}\right) P_{n}(\cos \psi) d \Omega^{\prime} .
\end{aligned}
$$

We now introduce the spherical lower-bound and upper-bound lateral density functions $\Gamma l_{n}$ and $\Gamma u_{n}$ of degree $n$ as

$$
\left.\begin{array}{l}
\Gamma l_{n}(\Omega) \\
\Gamma u_{n}(\Omega)
\end{array}\right\}=\frac{2 n+1}{4 \pi} \iint_{\Phi} \Delta \rho\left(\Omega^{\prime}\right) P_{n}(\cos \psi)\left\{\begin{array}{l}
D_{L}\left(\Omega^{\prime}\right) \\
D_{U}\left(\Omega^{\prime}\right)
\end{array} d \Omega^{\prime}=\sum_{m=-n}^{n} Y_{n, m}(\Omega)\left\{\begin{array}{l}
\Gamma l_{n, m} \\
\Gamma u_{n, m}
\end{array}\right.\right.
$$

where $Y_{n, m}$ are the (fully normalised) surface spherical harmonic functions. The higherorder terms $\left\{\Gamma l_{n}^{(i)}, \Gamma u_{n}^{(i)}: i=2,3,4, \ldots\right\}$ read

$$
\left.\begin{array}{l}
\Gamma l_{n}^{(i)}(\Omega) \\
\Gamma u_{n}^{(i)}(\Omega)
\end{array}\right\}=\frac{2 n+1}{4 \pi} \iint_{\Phi} \Delta \rho\left(\Omega^{\prime}\right) P_{n}(\cos \psi)\left\{\begin{array}{l}
D_{L}^{i}\left(\Omega^{\prime}\right) \\
D_{U}^{i}\left(\Omega^{\prime}\right)
\end{array} d \Omega^{\prime}=\sum_{m=-n}^{n} Y_{n, m}(\Omega)\left\{\begin{array}{l}
\Gamma l_{n, m}^{(i)} \\
\Gamma u_{n, m}^{(i)}
\end{array} .\right.\right.
$$

The coefficients $\Gamma l_{n, m}$ and $\Gamma u_{n, m}$ combine information on the geometry and lateral density distribution of a volumetric layer. Limiting the series up to the maximum degree of spherical harmonics $\bar{n}$ and inserting from Eqs.(10) and (11) to Eq.(9), we get

$$
V(r, \Omega)=4 \pi G R^{2} \sum_{n=0}^{\bar{n}}\left(\frac{R}{r}\right)^{n+1} \frac{1}{2 n+1} \sum_{m=-n}^{n}\left(F_{n, m}^{\Gamma l}-F_{n, m}^{\Gamma u}\right) Y_{n, m}(\Omega),
$$

where $F_{n, m}^{\Gamma l}$ and $F_{n, m}^{\Gamma u}$ are defined as follows 


$$
\begin{aligned}
F_{n, m}^{\Gamma l} \cong & \frac{\Gamma l_{n, m}}{R}-(n+2) \frac{\Gamma l_{n, m}^{(2)}}{2 R^{2}}+(n+2)(n+1) \frac{\Gamma l_{n, m}^{(3)}}{6 R^{3}}-(n+2)(n+1) n \frac{\Gamma l_{n, m}^{(4)}}{24 R^{4}} \\
& +(n+2)(n+1) n(n-1) \frac{\Gamma l_{n, m}^{(5)}}{120 R^{5}}+\sum_{k=5}^{n+2}\left(\begin{array}{c}
n+2 \\
k
\end{array}\right) \frac{(-1)^{k}}{k+1} \frac{\Gamma l_{n, m}^{(k+1)}}{R^{k+1}}
\end{aligned}
$$

and

$$
\begin{gathered}
F_{n, m}^{\Gamma u} \cong \frac{\Gamma u_{n, m}}{R}-(n+2) \frac{\Gamma u_{n, m}^{(2)}}{2 R^{2}}+(n+2)(n+1) \frac{\Gamma u_{n, m}^{(3)}}{6 R^{3}} \\
-(n+2)(n+1) n \frac{\Gamma u_{n, m}^{(4)}}{24 R^{4}}+(n+2)(n+1) n(n-1) \frac{\Gamma u_{n, m}^{(5)}}{120 R^{5}}+\sum_{k=5}^{n+2}\left(\begin{array}{c}
n+2 \\
k
\end{array}\right) \frac{(-1)^{k}}{k+1} \frac{\Gamma u_{n, m}^{(k+1)}}{R^{k+1}} .
\end{gathered}
$$

The gravitational attraction $g$ generated by the laterally varying density contrast layer with a variable depth and thickness is approximately defined as a negative radial derivative of the respective potential $V$. Hence

$$
g(r, \Omega) \cong-\frac{\partial V(r, \Omega)}{\partial r}=-G \iint_{\Phi} \Delta \rho\left(\Omega^{\prime}\right) \int_{R-D_{L}\left(\Omega^{\prime}\right)}^{R-D_{U}\left(\Omega^{\prime}\right)} \frac{\partial \ell^{-1}\left(r, \psi, r^{\prime}\right)}{\partial r} r^{\prime 2} \mathrm{~d} r^{\prime} d \Omega^{\prime} .
$$

By analogy with Eq.(12), the spectral representation of $g$ is defined in the following form

$$
g(r, \Omega)=4 \pi G R \sum_{n=0}^{\bar{n}}\left(\frac{R}{r}\right)^{n+2} \frac{n+1}{2 n+1} \sum_{m=-n}^{n}\left(F_{n, m}^{\Gamma l}-F_{n, m}^{\Gamma u}\right) Y_{n, m}(\Omega) .
$$

For a homogeneous density contrast layer with a variable depth and thickness, the density contrast $\Delta \rho$ is defined as the difference of the constant density values of the reference crust $\rho^{\text {crust }}$ and the constant density $\rho$ within the volumetric layer, i.e.

$$
\Delta \rho=\rho^{\text {crust }}-\rho, \quad\left(R-D_{U} \geq r^{\prime} \geq R-D_{L}\right) .
$$

The gravitational potential $V$ and attraction $g$ of an arbitrary homogeneous density contrast layer with a variable depth and thickness are then given by

$$
V(r, \Omega)=4 \pi G R^{2} \Delta \rho \sum_{n=0}^{\bar{n}}\left(\frac{R}{r}\right)^{n+1} \frac{1}{2 n+1} \sum_{m=-n}^{n}\left(F_{n, m}^{L}-F_{n, m}^{U}\right) Y_{n, m}(\Omega),
$$

and

$$
g(r, \Omega)=4 \pi G R \Delta \rho \sum_{n=0}^{\bar{n}}\left(\frac{R}{r}\right)^{n+2} \frac{n+1}{2 n+1} \sum_{m=-n}^{n}\left(F_{n, m}^{L}-F_{n, m}^{U}\right) Y_{n, m}(\Omega) .
$$

The numerical coefficients $F_{n, m}^{L}$ and $F_{n, m}^{U}$ in Eqs.(18) and (19) are defined as follows 


$$
F_{n, m}^{L} \cong \sum_{k=0}^{n+2}\left(\begin{array}{c}
n+2 \\
k
\end{array}\right) \frac{(-1)^{k}}{k+1} \frac{L_{n, m}^{(k+1)}}{R^{k+1}}, \quad F_{n, m}^{U} \cong \sum_{k=0}^{n+2}\left(\begin{array}{c}
n+2 \\
k
\end{array}\right) \frac{(-1)^{k}}{k+1} \frac{U_{n, m}^{(k+1)}}{R^{k+1}},
$$

where the coefficients $L_{n, m}$ and $U_{n, m}$ describe the geometry of the lower and upper bound of the homogeneous volumetric mass layer. The terms $\sum_{m=-n}^{n} L_{n, m} Y_{n, m}$ and $\sum_{m=-n}^{n} U_{n, m} Y_{n, m}$ define the spherical lower-bound and upper-bound functions $L_{n}$ and $U_{n}$ of degree $n$. They read

$$
\left.\begin{array}{c}
L_{n}(\Omega) \\
U_{n}(\Omega)
\end{array}\right\}=\frac{2 n+1}{4 \pi} \iint_{\Phi} P_{n}(\cos \psi)\left\{\begin{array}{l}
D_{L}\left(\Omega^{\prime}\right) \\
D_{U}\left(\Omega^{\prime}\right)
\end{array} d \Omega^{\prime}=\sum_{m=-n}^{n} Y_{n, m}(\Omega)\left\{\begin{array}{l}
L_{n, m} \\
U_{n, m}
\end{array} .\right.\right.
$$

The higher-order terms $\left\{L_{n}^{(i)}, U_{n}^{(i)}: i=2,3,4, \ldots\right\}$ are given by

$$
\left.\begin{array}{c}
L_{n}^{(i)}(\Omega) \\
U_{n}^{(i)}(\Omega)
\end{array}\right\}=\frac{2 n+1}{4 \pi} \iint_{\Phi} P_{n}(\cos \psi)\left\{\begin{array}{l}
D_{L}^{i}\left(\Omega^{\prime}\right) \\
D_{U}^{i}\left(\Omega^{\prime}\right)
\end{array} d \Omega^{\prime}=\sum_{m=-n}^{n} Y_{n, m}(\Omega)\left\{\begin{array}{l}
L_{n, m}^{(i)} \\
U_{n, m}^{(i)}
\end{array} .\right.\right.
$$

\section{NUMERICAL EXAMPLES}

The expressions derived in Section 2 were utilised to calculate the gravitational field generated by the major known density contrast structures within the Earth's crust (excluding the ocean density contrast) beneath the geoid surface. The $2 \times 2$ arc-deg discrete data of thickness, depth, and density of the (soft and hard) sediments and the (upper, middle, and lower) consolidated crust components from CRUST2.0 were used to generate the sets of coefficients $\Gamma l_{n, m}$ and $\Gamma u_{n, m}$ which describe the spatial distribution of crustal density. The computation of $\Gamma l_{n, m}$ and $\Gamma u_{n, m}$ was done according to Eqs.(10) and (11) by applying the spherical harmonic analysis of thickness, depth, and lateral density data.

The coefficients $\Gamma l_{n, m}$ and $\Gamma u_{n, m}$ with a spectral resolution complete to degree 90 of spherical harmonics were used to compute the gravitational potential and attraction generated by the sediments and consolidated crust components density contrasts (by applying methods for a spherical harmonic synthesis). All computations were realised globally on a $1 \times 1$ arc-deg geographical grid at the Earth's surface. The density contrast was defined relative to the reference crustal density of $2670 \mathrm{kgm}^{-3}$ (cf. Hinze, 2003). We note here that the choice of the reference crustal density is optional depending on a particular purpose of the numerical study. Moreover, when the objective is to study the sub-crustal density distribution anomalies, then the density contrast of the crustal components is usually taken relative to the average density of the lithospheric mantle (upper mantle). 


\section{R. Tenzer et al.}

The gravitational field quantities generated by the sediments density contrast are shown in Figs. 1 and 2. The potential is everywhere positive and globally varies between 1629 and $3138 \mathrm{~m}^{2} \mathrm{~s}^{-2}$ with the mean of $2249 \mathrm{~m}^{2} \mathrm{~s}^{-2}$, and the standard deviation is $377 \mathrm{~m}^{2} \mathrm{~s}^{-2}$. The corresponding attraction varies from 12 to $122 \mathrm{mGal}$, with the mean of $34 \mathrm{mGal}$, and the standard deviation is $19 \mathrm{mGal}$. The maxima of computed gravitational

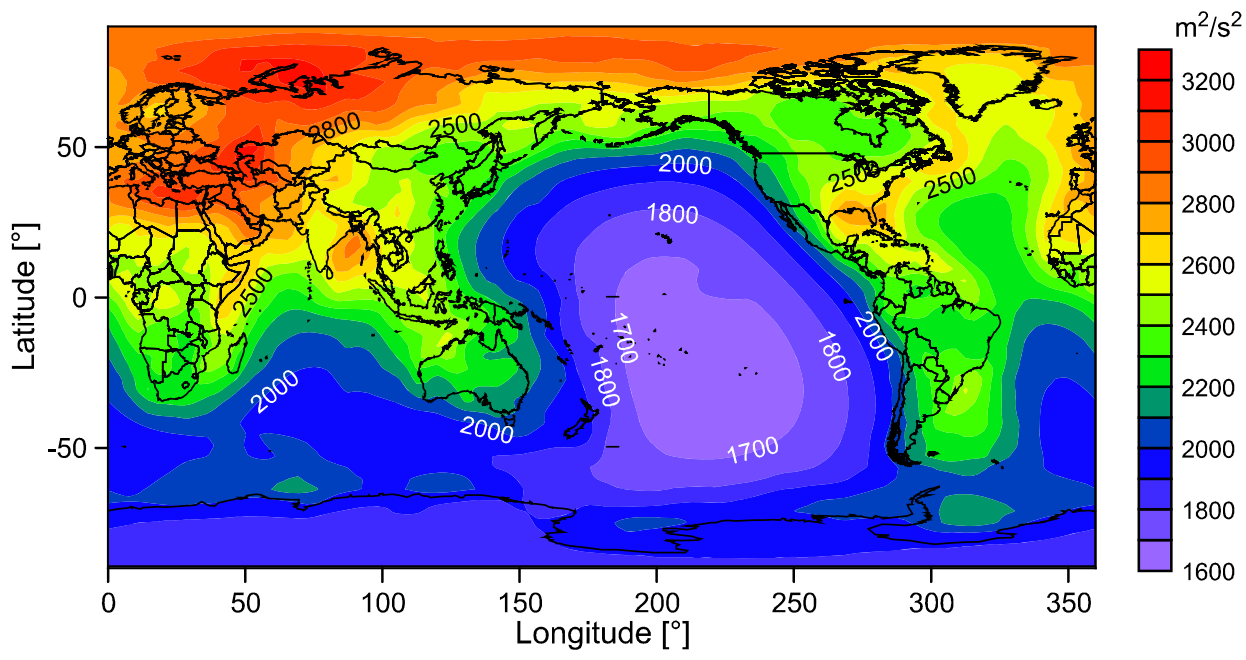

Fig. 1. The gravitational potential due to the sediments density contrast computed globally with a spectral resolution complete to spherical harmonic degree 90 .

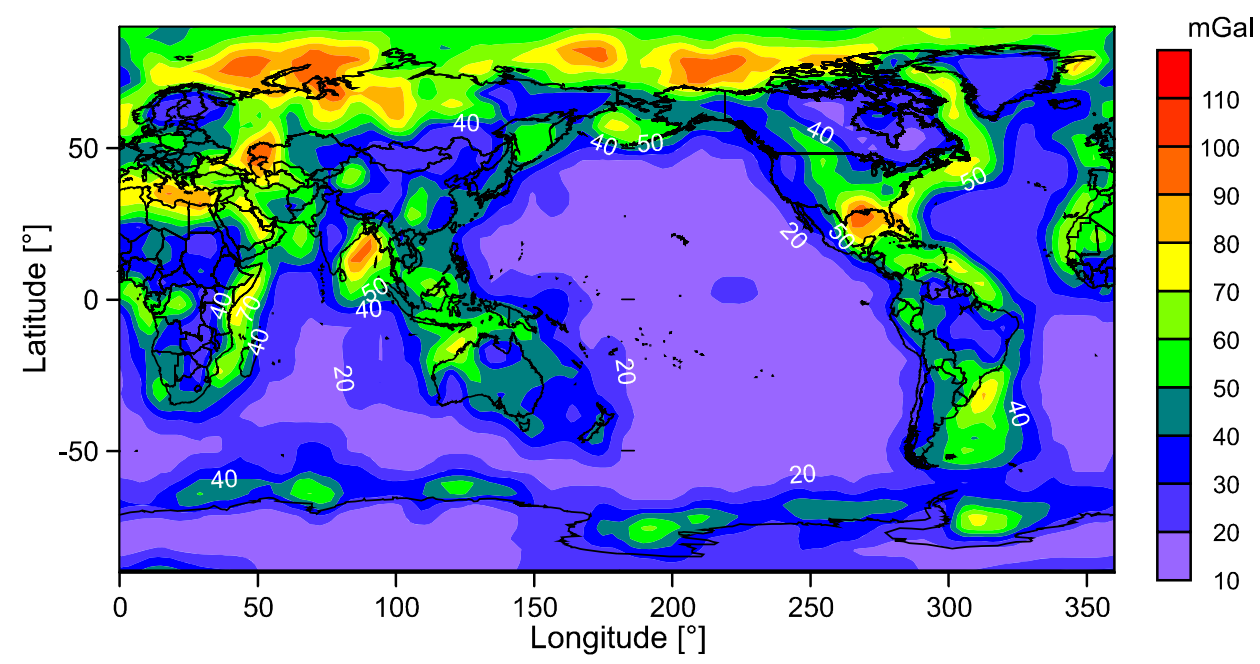

Fig. 2. The gravitational attraction due to the sediments density contrast computed globally with a spectral resolution complete to spherical harmonic degree 90 . 
field quantities were found along continental shelf regions with the largest sediment deposits. The positive gravitational field values are due to the fact that the range of the CRUST2.0 sediment densities is between 1700 and $2600 \mathrm{kgm}^{-3}$. The sediment density is thus below the adopted reference crustal density of $2670 \mathrm{kgm}^{-3}$, and the respective sediments density contrast is always positive.

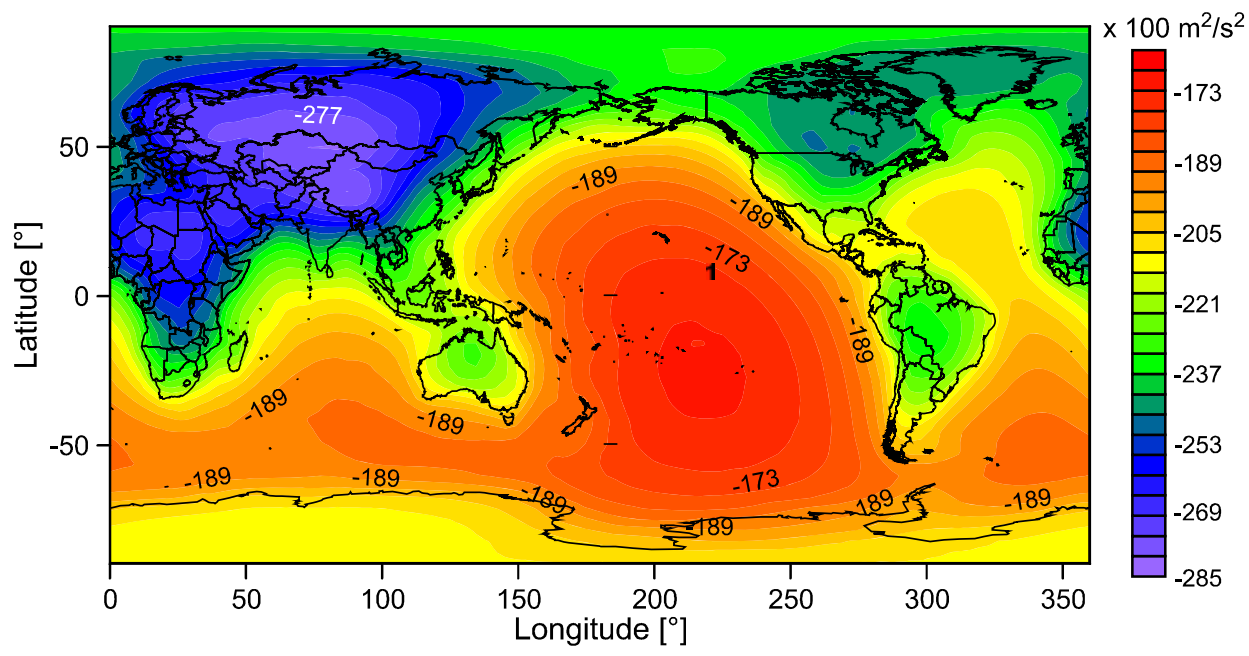

Fig. 3. The gravitational potential due to the consolidated crust density contrast computed globally with a spectral resolution complete to spherical harmonic degree 90.

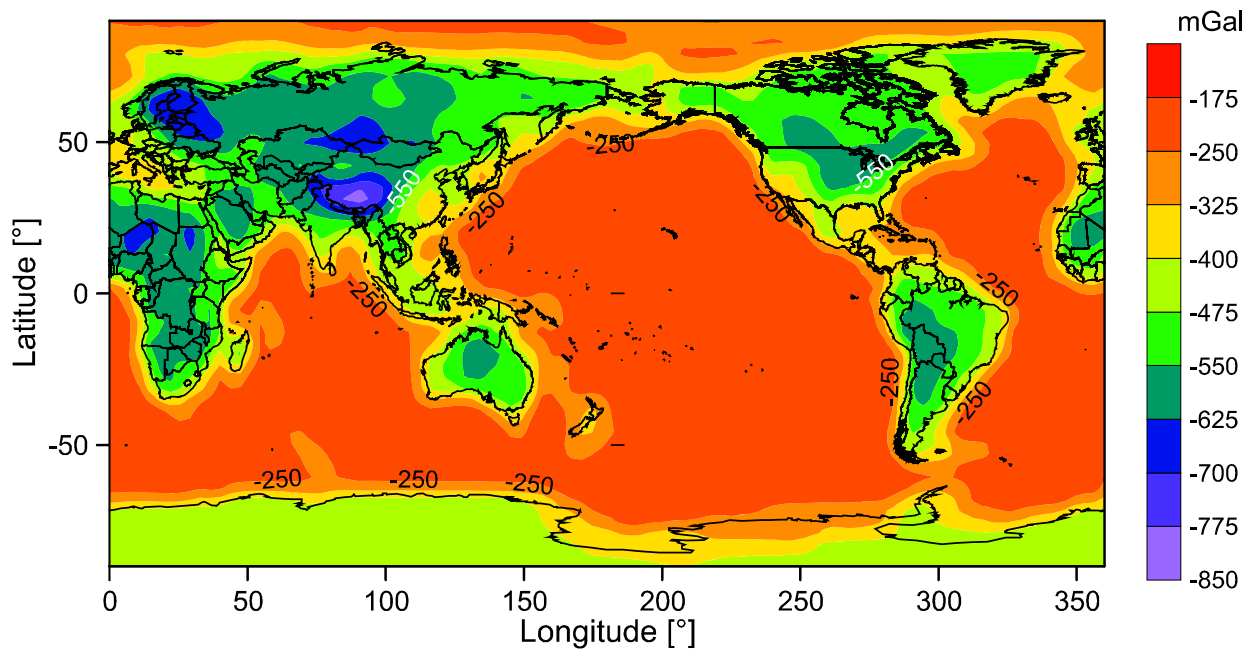

Fig. 4. The gravitational attraction due to the consolidated crust density contrast computed globally with a spectral resolution complete to spherical harmonic degree 90 . 
The gravitational field quantities generated by the consolidated crust density contrast were computed individually for the CRUST2.0 upper, middle, and lower crustal components. Their complete gravitational contributions are shown in Figs. 3 and 4. The potential is everywhere negative and globally varies from -27986 to $-16381 \mathrm{~m}^{2} \mathrm{~s}^{-2}$ with the mean of $-21126 \mathrm{~m}^{2} \mathrm{~s}^{-2}$, and the standard deviation is $2925 \mathrm{~m}^{2} \mathrm{~s}^{-2}$. The corresponding attraction varies from -824 to $-184 \mathrm{mGal}$ with the mean of $-338 \mathrm{mGal}$, and the standard deviation is $137 \mathrm{mGal}$. The crust components density contrast is mostly negative for the range of crust densities between 2600 and $3100 \mathrm{kgm}^{-3}$.

\section{CONCLUSIONS}

We have derived the expressions for computing the gravitational potential and its radial derivative generated by the homogeneous and laterally varying mass density contrast layers with a variable depth and thickness using the spherical harmonic analysis and synthesis of gravity field. The expressions for the homogeneous mass density contrast layer utilise the spherical lower-bound and upper-bound spherical functions $L_{n}$ and $U_{n}$ which describe the geometry of a particular volumetric mass layer of homogeneous density. The corresponding expressions for the laterally varying mass density contrast layer utilise the spherical lower-bound and upper-bound lateral density functions $\Gamma l_{n}$ and $\Gamma u_{n}$. These functions combine the information on the geometry and lateral density distribution of the volumetric mass layer.

In numerical examples, we have used the CRUST2.0 density, thickness, and depth data of sediments and consolidated crust components to generate the coefficients $\Gamma l_{n, m}$ and $\Gamma u_{n, m}$ for representing the global crust density structures beneath the geoid surface in the spectral domain. These coefficients were then used for computing the corresponding gravitational field quantities with a low spectral resolution complete to degree 90 of spherical harmonics. The results revealed that the largest gravitational signal is due to the variable geological structures within the continental crust with large thickness. The largest gravitational signal due to the sediments density contrast was found mostly along the continental shelf regions. The absolute maxima of the gravitational field quantities generated by the sediments density contrast are several times smaller than the maxima of the gravitational field quantities generated by the remaining crust density contrast structures.

We anticipate large errors in the computed gravitational field. These errors are attributed mainly to the heterogeneities of the consolidated crust (especially over continental crust) and the Moho uncertainty (especially under significant orogens). A realistic assessment of these errors is not simple. Kaban et al. (2003) estimated, for instance, that the errors in computed values of the gravitational attraction can reach as much as $100 \mathrm{mGal}$ over continental regions, while about $40 \mathrm{mGal}$ over the oceanic areas. It corresponds to a relative inaccuracy of about $10 \%$. Similar relative errors are expected in computed values of the gravitational potential. 
Acknowledgments: Pavel Novák was supported by the Project MSM4977751301 of the Czech Ministry of Education, Youth and Sport. Peter Vajda was supported by the Slovak Research and Development Agency under the contract No. APVV-0194-10 and by Vega grant agency under project No. 2/0107/09.

\section{References}

Artemjev M.E., Kaban M.K., Kucherinenko V.A., Demjanov G.V. and Taranov V.A., 1994. Subcrustal density inhomogeneities of the Northern Euroasia as derived from the gravity data and isostatic models of the lithosphere. Tectonophysics, 240, 248-280.

Bassin C., Laske G. and Masters G., 2000. The current limits of resolution for surface wave tomography in North America. EOS Trans AGU, 81, F897.

Čadek O. and Martinec Z., 1991. Spherical harmonic expansion of the earth's crustal thickness up to degree and order 30. Stud. Geophys. Geod., 35, 151-165.

Eshagh M., 2009. The effect of lateral density variations of crustal and topographic masses on GOCE gradiometric data - A study in Iran and Fennoscandia. Acta Geod. Geophys. Hung., 44, 399-418.

Hinze W.J., 2003. Bouguer reduction density, why 2.67? Geophysics, 68, 1559-1560.

Hobson E.W., 1931. The Theory of Spherical and Ellipsoidal Harmonics. Cambridge University Press, Cambridge, U.K.

Kaban M.K., Schwintzer P., Artemieva I.M. and Mooney W.D., 2003. Density of the continental roots: compositional and thermal contributions. Earth Planet. Sci. Lett., 209, 53-69.

Meier U., Curtis A. and Trampert J., 2007. Global crustal thickness from neural network inversion of surface wave data. Geophys. J. Int., 169, 706-722.

Mooney W.D., Laske G. and Masters T.G., 1998. CRUST 5.1: A global crustal model at $5^{\circ} \times 5^{\circ}$. J. Geophys. Res., 103(B), 727-747.

Moritz H., 1980. Advanced Physical Geodesy. Abacus Press, Tunbridge Wells.

Rummel R., Rapp R.H., Suenkel H. and Tscherning C.C., 1988. Comparison of Global Topographic/Isostatic Models to the Earth's Observed Gravitational Field. Report 388. The Ohio State University, Columbus, Ohio.

Shapiro N.M. and Ritzwoller M.H., 2002. Monte-Carlo inversion for a global shear-velocity model of the crust and upper mantle. Geophys. J. Int., 151, 88-105.

Sjöberg L.E. and Nahavandchi H., 2000. The atmospheric geoid effects in Stokes formula. Geophys. J. Int., 140, 95-100.

Sjöberg L.E., 1998. The exterior Airy/Heiskanen topographic-isostatic gravity potential anomaly and the effect of analytical continuation in Stokes' formula. J. Geod. 72: 654-662.

Sun W. and Sjöberg L.E., 2001. Convergence and optimal truncation of binomial expansions used in isostatic compensations and terrain corrections. J. Geodesy, 74, 627-636.

Tenzer R., Hamayun and Vajda P., 2009. Global maps of the CRUST 2.0 crustal components stripped gravity disturbances. J. Geoph. Res., 114, B05408. 
Tenzer R., Abdalla A., Vajda P. and Hamayun, 2010. The spherical harmonic representation of the gravitational field quantities generated by the ice density contrast. Contrib. Geophys. Geod., 40, 207-223.

Tenzer R., Novák P. and Gladkikh V., 2011. On accuracy of the bathymetry-generated gravitational field quantities for a depth-dependent seawater density distribution. Stud. Geophys. Geod., 55, DOI: $10.1007 / \mathrm{s} 11200-010-0074-y$ (in print). 